\title{
Non-alcoholic fatty liver disease: need for a balanced nutritional source
}

\author{
Jayagopalan Veena ${ }^{1}$, Anjaneyulu Muragundla ${ }^{1}$, Srinivas Sidgiddi ${ }^{2 *}$ and Swaminathan Subramaniam ${ }^{2}$ \\ ${ }^{1}$ Syngene International Limited, Biocon Park, Plot No. 2E3, Bommasandra IV Phase, Jigani Link Road, \\ Bangalore 560 099, India \\ ${ }^{2}$ Clinical Research and Nutrition Science, Abbott Nutrition Research and Development (ANRD), Syngene International \\ Limited, Biocon Park, Plot No. 2\&3, Bommasandra IV Phase, Jigani Link Road, Bangalore 560 099, India
}

(Submitted 28 March 2014 - Final revision received 21 July 2014-Accepted 24 July 2014 - First published online 2 October 2014)

\begin{abstract}
Non-alcoholic fatty liver disease (NAFLD) and non-alcoholic steatohepatitis (NASH) are an increasingly common chronic liver disease closely associated with diabetes and obesity that have reached epidemic proportions. Reports on the prevalence of NAFLD have suggested that $27-34 \%$ of the general population in the USA and $40-90 \%$ of the obese population worldwide have this disease. Increasing urbanisation rate and associated inappropriate lifestyle changes are not only the risk factors of diabetes, but also unmask genetic predisposition in various populations for the metabolic syndrome and its manifestations including NAFLD and NASH. Lifestyle modifications and balanced nutrition are among the foremost management strategies along with ursodeoxycholic acid, metformin, vitamin $\mathrm{E}$ and pentoxifylline. Although weight reduction associated with current therapeutic strategies has shown some promise, maintaining it in the long run is largely unsuccessful. With the safety of pharmacotherapy still being uncertain and can be started only after confirmation, other reasonable interventions such as nutrition hold promise in preventing disease progression. The role of dietary components including branchedchain amino acids, methionine, choline and folic acid is currently being evaluated in various clinical trials. Nutritional approaches sought to overcome the limitations of pharmacotherapy also include evaluating the effects of natural ingredients, such as silymarin and spirulina, on liver disease. Understanding the specific interaction between nutrients and dietary needs in NAFLD and maintaining this balance through either a diet or a nutritional product thus becomes extremely important in providing a more realistic and feasible alternative to treat NAFLD. A planned complete nutritional combination addressing specific needs and helping to prevent the progression of NAFLD is the need of the hour to avert people from ending up with complications.
\end{abstract}

Key words: Non-alcoholic fatty liver disease: Non-alcoholic steatohepatitis: Nutrition: Diabetes: Cirrhosis: Obesity: Balanced diet

Non-alcoholic fatty liver disease (NAFLD), the term introduced by Ludwig et $a l^{(1)}$, refers to a spectrum of steatotic liver disease in the absence of alcohol consumption in amounts considered to be harmful to the liver. The spectrum histologically characterised by macrovesicular and microvesicular hepatic steatosis is a major cause of liver-related morbidity and mortality ${ }^{(2)}$. NAFLD also described as non-alcoholic steatonecrosis $^{(3)}$, fatty liver hepatitis ${ }^{(4)}$ and non-alcoholic fatty hepatitis ${ }^{(5)}$ is now recognised as a major health burden with its common occurrence in the general population and an unwarranted potential to progress to a more severe form called non-alcoholic steatohepatitis (NASH), then to fibrosis, cirrhosis and, finally, leading to liver failure ${ }^{(6)}$. It is also regarded as the leading cause of cryptogenic cirrhosis ${ }^{(7)}$. Being the most common liver disease in developing and developed nations, NAFLD have been generally associated with obesity and the metabolic syndrome (MetS; hypertension, dyslipidaemia, increased waist circumference and insulin resistance (IR)) in earlier studies ${ }^{(8)}$. However, its occurrence is not restricted to individuals with the MetS. Population studies have reported its occurrence even in non-obese/ diabetic $^{(6)}$ individuals and in children ${ }^{(8)}$. The prevalence of NAFLD has been found to be higher in males and increases with the progression of age ${ }^{(9)}$. The major limiting factor in curtailing NAFLD is the absence of signs and symptoms in addition to limited sensitive and specific diagnostic tests. Since its underlying mechanisms are poorly understood, the efficacy and safety of pharmacotherapy in the treatment of NAFLD remains uncertain. Lifestyle changes including physical activity (PA) and diet have often been the first step of therapy ${ }^{(10)}$.

Abbreviations: ALT, alanine aminotransferase; AMPK, 5'-AMP-activated protein kinase; AST, aspartate aminotransferase; BCAA, branched-chain amino acids; IR, insulin resistance; MAT, methionine adenosyltransferase; MetS, metabolic syndrome; NAFLD, non-alcoholic fatty liver disease; NASH, non-alcoholic steatohepatitis; PA, physical activity; SAMe, S-adenosylmethionine.

*Corresponding author: Dr S. Sidgiddi, email srinivas.sidgiddi@abbott.com 
Although the role of nutrition in the management of NAFLD is rather complex, with the interplay of nutrients, it still serves as one of the major route for preventing the progression of the disease, and is a promising mode of treatment.

Data on the prevalence of NAFLD across the globe are diverging depending on the definitions, lifestyle habits, differences in the population studied and the method used for diagnosis. The occurrence of NAFLD in the general population has been found to be about $27-34 \%$ in the USA, $20-30 \%$ in Europe, $20-40 \%$ in Western countries, $20-30 \%$ in the Middle East, $15 \%$ in the Far East, $9-30 \%$ in India and $18 \%$ in Pakistan ${ }^{(11)}$. The prevalence of NAFLD has been estimated in $40-90 \%$ of the obese population worldwide. NAFLD has been diagnosed not only in the adult population but also among children. According to the statistics, an estimate of $13-14 \%$ of the paediatric population in the USA and $2 \cdot 6-10 \%$ in Europe has NAFLD. It is estimated that by the year 2015, three-quarters of the world's 300 million adults with diabetes will be in non-industrialised countries, and almost one-third in India and China alone. It is estimated that $30-90 \%$ of the diabetic population and $15-20 \%$ of the obese population in India have NAFLD ${ }^{(12)}$. At least $10-20 \%$ of the general population has been found to progress to NASH and $40-55 \%$ of the patients at tertiary care centres have shown the prevalence of $\mathrm{NASH}^{(13)}$. All these recent data suggest that the condition of NAFLD is increasing worldwide in the recent past. This cries for a detailed study of the incidence and prevalence of NAFLD that is closely associated with the MetS. Moreover, untreated steatosis progresses to more advanced fibrosis and then to cirrhosis, a stage at which it becomes irreversible ${ }^{(14)}$. Thus, there is a need to deal with the disease at a stage where it is still responsive and reversible. This can be achieved to a large extent by successful nutritional management. There is an immediate need to address this issue in the general population, generate data on the rate of prevalence, educate about the need to control the progression of the disease with suitable lifestyle modifications, and address the required nutritional needs before it is too late.

\section{The spectrum - risk factors and their progression}

NAFLD is defined as a condition in which there is excessive fat accumulation in the form of TAG (steatosis) in the liver (>5\% of hepatocytes histologically). It has been broadly classified into two types: primary (associated with IR or the MetS) and secondary (caused by the intake of some drugs, surgery or total parenteral nutrition $)^{(6)}$. Irrespective of the type, $40 \%$ of the patients progress to $\mathrm{NASH}^{(15)}$, with the major manifestation being the increased number of apoptotic and necrotic hepatocytes in the liver ${ }^{(16)}$. The exact cause of $\mathrm{NASH}$ is not yet been elucidated, and it may not be the same in every patient. However, in general, this transformation underlies increased lipid peroxidation, inflammation and increased production of oxidative radicals. This condition is virtually indistinguishable histologically from the condition of alcoholic steatohepatitis ${ }^{(17)}$. When left untreated, 74\% of the patients with NASH end up with fibrosis of the liver, ultimately leading to cirrhosis ${ }^{(18)}$ (Fig. 1). The most prevalent risk factors associated with NAFLD are obesity, increased fasting glucose and IR. In addition, central obesity and high BMI, hypertension ${ }^{(8)}$, longer duration of diabetes, age above 40 years $^{(19)}$, high serum levels of liver enzymes, TAG, and decreased antioxidant vitamins, $\mathrm{Ca}$ and vitamin $\mathrm{D}^{(20)}$ have also been implicated in the development and progression of NAFLD when left untreated.

\section{Pathogenesis of non-alcoholic fatty liver disease}

Under normal metabolic conditions, lipolysis in the adipose tissue releases NEFA into the blood stream, which reach liver hepatocytes for oxidation. Fat molecules undergo oxidation in the mitochondria. Glucose and TAG from the diet also contribute to NEFA content in the liver by the process of de novo lipogenesis. Apart from being oxidised, NEFA is also stored as TAG in the liver, which is transported by VLDL, thus contributing to the export of FA (Fig. 2). The controlled occurrence of these activities in consortium maintains the levels of NEFA in the liver and serum, thus preventing the formation of fatty liver. Any discrepancy in this complex balance of events could lead to a fatty liver, ultimately resulting in fibrosis (Fig. 3).

\section{Non-alcoholic fatty liver disease as a fundamental component of the metabolic syndrome}

IR is often considered as a key player in the pathogenesis of NAFLD, to an extent that NAFLD is even considered as a hepatic component of IR or the MetS ${ }^{(21,22)}$. Although the underlying mechanisms of NAFLD are in the early stages of being unravelled, it mostly appears as an interaction, more specifically an imbalance between adipokines and cytokines that alter insulin sensitivity in insulin-targeted organs, including the liver. Induction typically involves two consecutive steps: widely called as the 'two-hit hypothesis', whereby the first 'hit' - steatosis - sensitises the liver to a variety of second 'hits' that lead to necro-inflammation and fibrosis ${ }^{(23)}$ The initial hit leading to the development of a fatty liver renders hepatocytes susceptible to the subsequent multiple hits including adipokine interactions, lipid peroxidation, oxidative stress, endotoxinaemia, hepatic stellate cell activation and mitochondrial dysfunction. Inflammatory parameters such as $\mathrm{TNF} \alpha$ can activate the translocation of NF- $\mathrm{BB}$ into the nucleus, resulting in the production of reactive oxygen molecules that could aggravate the inflammation of stellate cells and lead to fibrosis and cell death. Increased levels of serum fatty acids and hydrophobic bile acids lead to impaired mitochondrial function $^{(24)}$. Extra-mitochondrial fatty acid oxidation in the endoplasmic reticulum and peroxisomes results in the release of reactive oxygen species that further contribute to inflammation and collagen deposition, leading to fibrosis ${ }^{\text {(25) }}$ (Fig. 3).

Adiponectin, a polypeptide synthesised and released by adipocytes in relatively high quantities, is characterised by potent anti-inflammatory, antidiabetic, antiatherogenic and insulin-sensitising properties. Conversely, low levels of serum adiponectin are observed in individuals with IR and 


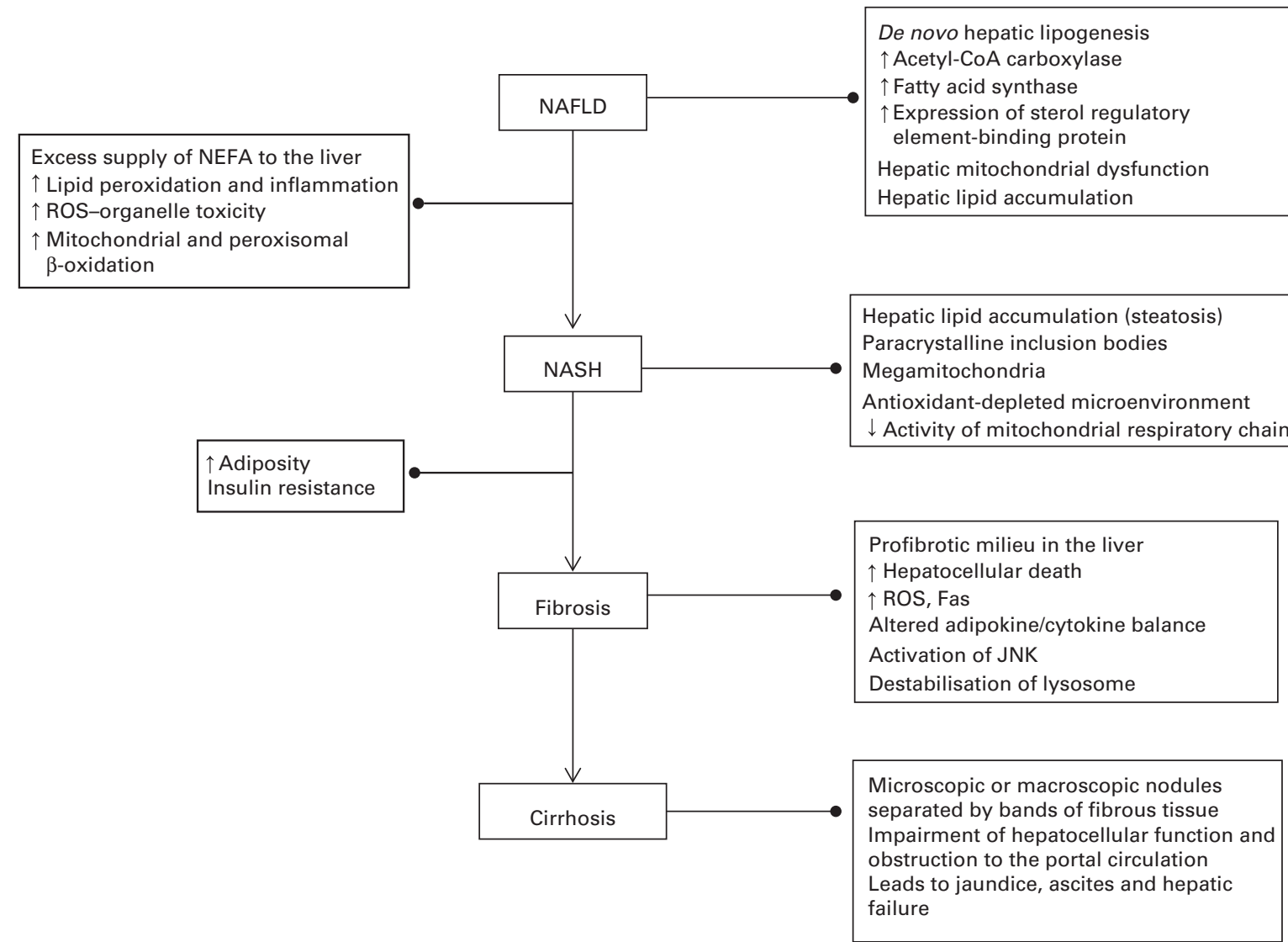

Fig. 1. Spectrum of non-alcoholic fatty liver disease (NAFLD). Changes associated with each stage from NAFLD to cirrhosis of the liver are illustrated. JNK, c-Jun $\mathrm{N}$-terminal kinase; ROS, reactive oxygen species; $\mathrm{NASH}$, non-alcoholic steatohepatitis.

the MetS ${ }^{(25)}$. Adiponectin released by adipocytes activates 5 -AMP-activated protein kinase (AMPK) and PPAR through the adiponectin receptor 2 transmembrane receptor. The activation of AMPK and PPAR has a positive downstream effect on insulin sensitivity. Their activation, in turn, induces the expression of genes involved in increased fatty acid oxidation, increased NEFA influx, decreased gluconeogenesis and decreased de novo lipogenesis. Increased accumulation of fat mass due to increased energy consumption and decreased energy combustion leads to decreased levels of adiponectin and increased levels of inflammatory cytokines. This decreases insulin-sensitising actions in hepatocytes and renders the organ more prone to become a fatty liver ${ }^{(26)}$.

\section{Diagnosis}

A detailed review of recommendations suggesting preferred approaches to the diagnostic, therapeutic and preventive aspects of care has been described elsewhere ${ }^{(27,28)}$. A brief clinical pathway is described here (which is not currently part of any standard algorithm). To date, there are no definite tests exclusively for the detection of NAFLD, and the symptomfree nature of the condition makes the diagnosis even more challenging. Another concerning aspect of the diagnosis is to decipher whether the patients are presenting simple steatosis or NASH, and more importantly, the stage of fibrosis.
Recently, serum analysis and liver biopsy carried out in patients with NASH showed lower levels of circulating serum B-cell lymphoma 2, decreased IR and $\gamma$-glutamyl-transpeptidase, and significantly more apoptotic hepatocytes ${ }^{(29)}$. Gene profiling of liver biopsies from patients with mild and severe NAFLD has identified specific gene patterns associated with

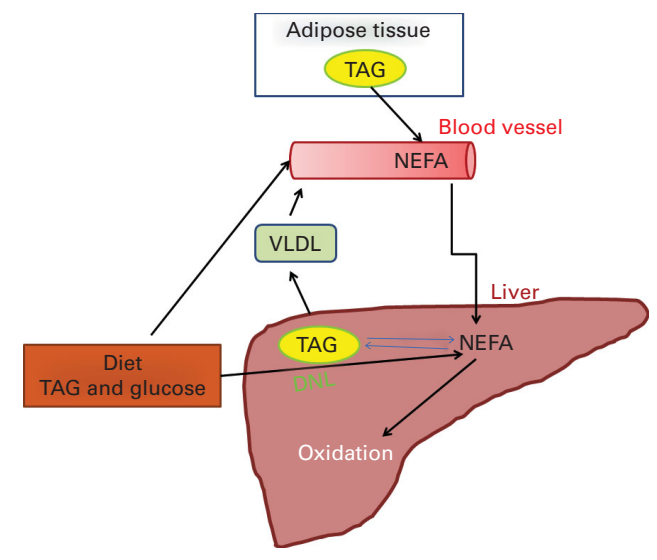

Fig. 2. Normal metabolism of the liver: TAG from adipose tissue enters into the circulation as NEFA, which is taken up by the liver. They are further metabolised by $\beta$-oxidation in the mitochondria. NEFA are converted to TAG in the liver, which are carried by VLDL back into the blood flow. Diet also contributes to the TAG load in the liver. Glucose from the diet is converted to NEFA in hepatocytes by the process of de novo lipogenesis (DNL). 


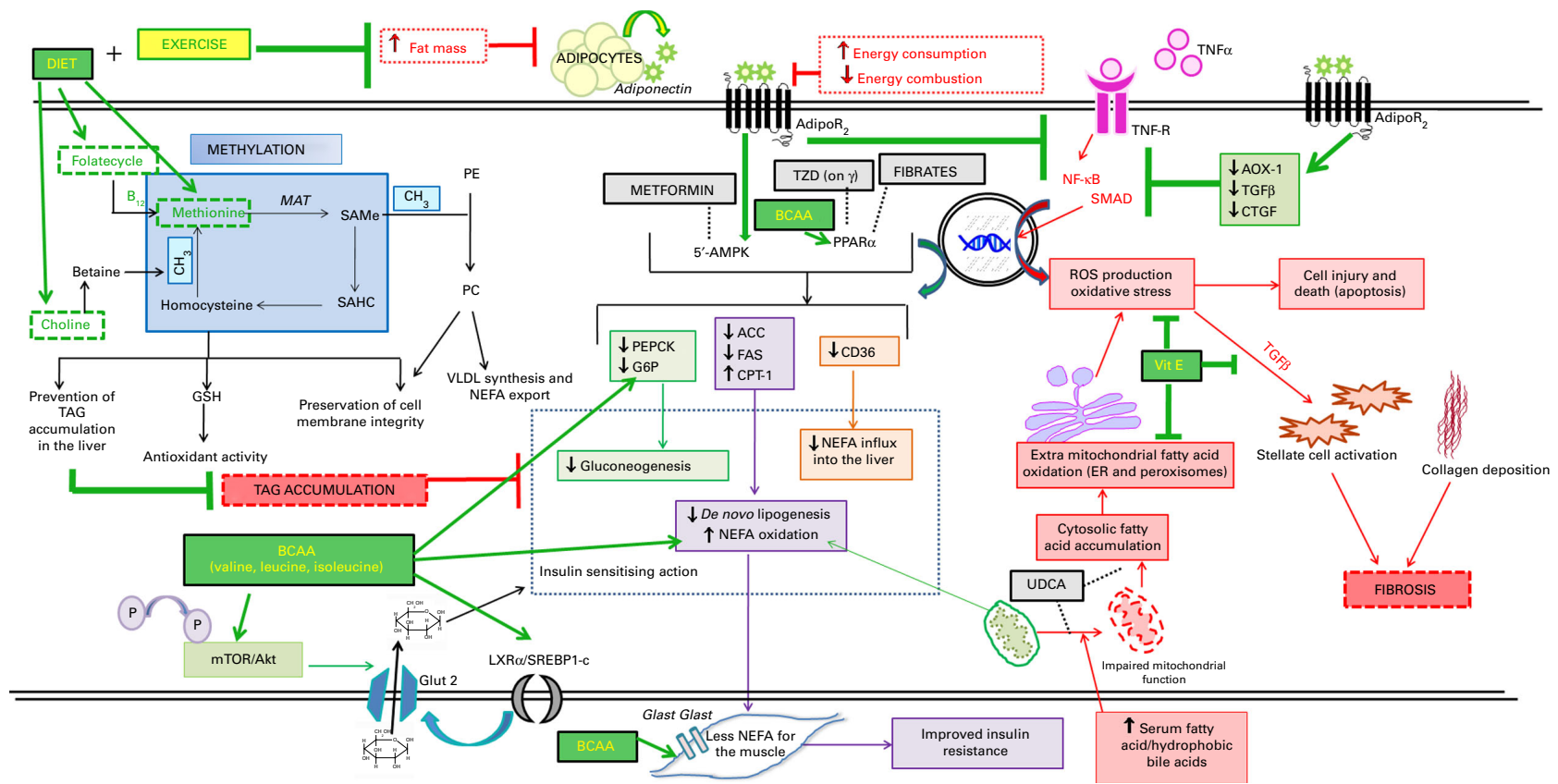

Fig. 3. Disease progression, possible mechanisms and targets. Increased levels of serum fatty acids and hydrophobic bile acids impair the mitochondrial function of oxidation. The extra mitochondrial oxidation of fatty acids in the endoplasmic reticulum (ER) and peroxisomes results in oxidative stress. Increased levels of reactive oxygen species (ROS) lead to the activation of inflammatory stellate cells and the deposition of collagen fibres, which collectively lead to fibrosis and cell death. Activation of NF-kB and translocation of Smad into the nucleus also activates the oxidative stress pathway. The methylation reaction in hepatocytes results in the prevention of TAG accumulation, synthesis of glutathione (GSH) which has antioxidant properties, and preservation of cell membrane integrity. A deficiency in methyl donors including folic acid, methionine and choline or a genetic condition in which methionine adenosyl transferase (MAT) is not synthesised in appropriate quantities results in the accumulation of TAG, increased oxidative stress and unstable cell membrane. Accumulation of TAG can result in insulin resistance. Methyl donors are obtained mainly from the diet. Branched-chain amino acids (BCAA) can act on PPAR $\alpha$, decrease glucose-6-phosphatase (G6P), increase NEFA oxidation, increase glucose uptake via the activation of liver X receptor (LXR) $\alpha$ or sterol regulatory element-binding protein 1 (SREBP1) receptor and, in turn, increase insulin sensitivity. Leucine acts on the mammalian target of rapamycin (mTOR)/Akt pathway that activates downstream events favouring glucose uptake by hepatocytes. BCAA are also obtained by a balanced nutrition. The activation of oxidative stress pathways is prevented to a large extent by vitamin $\mathrm{E}$. Under normal conditions, adipocytes release adiponectin, which binds to the adiponectin receptor (AdipoR $)_{2}$ and activates the AMP-activated protein kinase (AMPK) and PPAR pathways. Downstream effects include decreased levels of phosphoenolpyruvate carboxykinase (PEPCK), G6P, acetyl CoA carboxylase (ACC) and fatty acid synthase (FAS) activities and inducing carnitine palmitoyltransferase 1 (CPT-1) activity, thereby decreasing gluconeogenesis, increasing NEFA oxidation and decreasing de novo lipogenesis. The influx of NEFA into the liver is decreased by down-regulating the expression of the hepatic protein CD36. Adiponectin also prevents the formation of fibrosis by down-regulating the expression of aldehyde oxidase (AOX) -1 , transforming growth factor- $\beta$ and connective tissue growth factor. It also prevents the translocation of Smad2 and NF-кB into the nucleus, thus preventing the oxidative stress cascade. Increased consumption of energy without their expenditure prevents the positive effects of adiponectin. Different targets of currently used pharmacological agents are also indicated. Since pharmacological agents are usually sought for after a confirmation of liver biopsy, it is indeed important to note that nutritional components also target similar pathways and can be administered at the very early stage of the disease with least risk involved. PE, phosphatidyl ethanolamine; SAMe, S-adenosylmethionine; SAHC, S-adenosylhomocysteine; PC, phosphatidyl choline; TZD, thiozolidinedione; UDCA, ursodeoxycholic acid; Vit E, vitamin E.

severe NAFLD ${ }^{(30)}$, and these could be exploited in future to improve the diagnosis and treatment. The most widely used non-invasive tests are ultrasonography and plasma aminotransferase levels (aspartate aminotransferase (AST)/alanine aminotransferase (ALT)). Briefly, when an increased level of ALT is detected, causes related to alcohol use or viral infections are excluded. Patients are then tested for clinical signs of IR including obesity, dyslipidaemia, hypertension and type 2 diabetes mellitus. If the results are positive, the patients are either tested for fat accumulation using the principles of ultrasonography and then advised for primary care management protocols such as body-weight reduction and physical exercise in the daily routine, or directly directed towards primary care management without using ultrasonography. Based on the degree of improvement of insulin sensitivity, patients are treated for the risk factors of NAFLD including diabetes, obesity and hypertension. Occasionally, patients are subjected to the NAFLD fibrosis score, and those with a low likelihood score are directed towards primary management care. In contrast, patients showing persistence of risk factors and at-risk patients who show very high levels of ALT are subjected to ultrasonography. Depending on NAFLD fibrosis scores or ALT levels, patients are further advised for transient elastography or for liver biopsy. Based on the histopathological profile of liver biopsy tissue, patients are enrolled in clinical trials for treatment or registered for a liver transplant (Fig. 4)

Most of the diagnosis of NAFLD is largely based on ALT levels; however, there is a disadvantage that these levels are not likely to be sensitive enough. Burgert et al. ${ }^{(31)}$ found that only $48 \%$ of NAFLD patients had abnormal ALT levels, thus missing out approximately $50 \%$ of the population with NAFLD, while Amarapurkar \& Patel ${ }^{(32)}$ found histological evidence of cirrhosis in $23 \%$ of their 


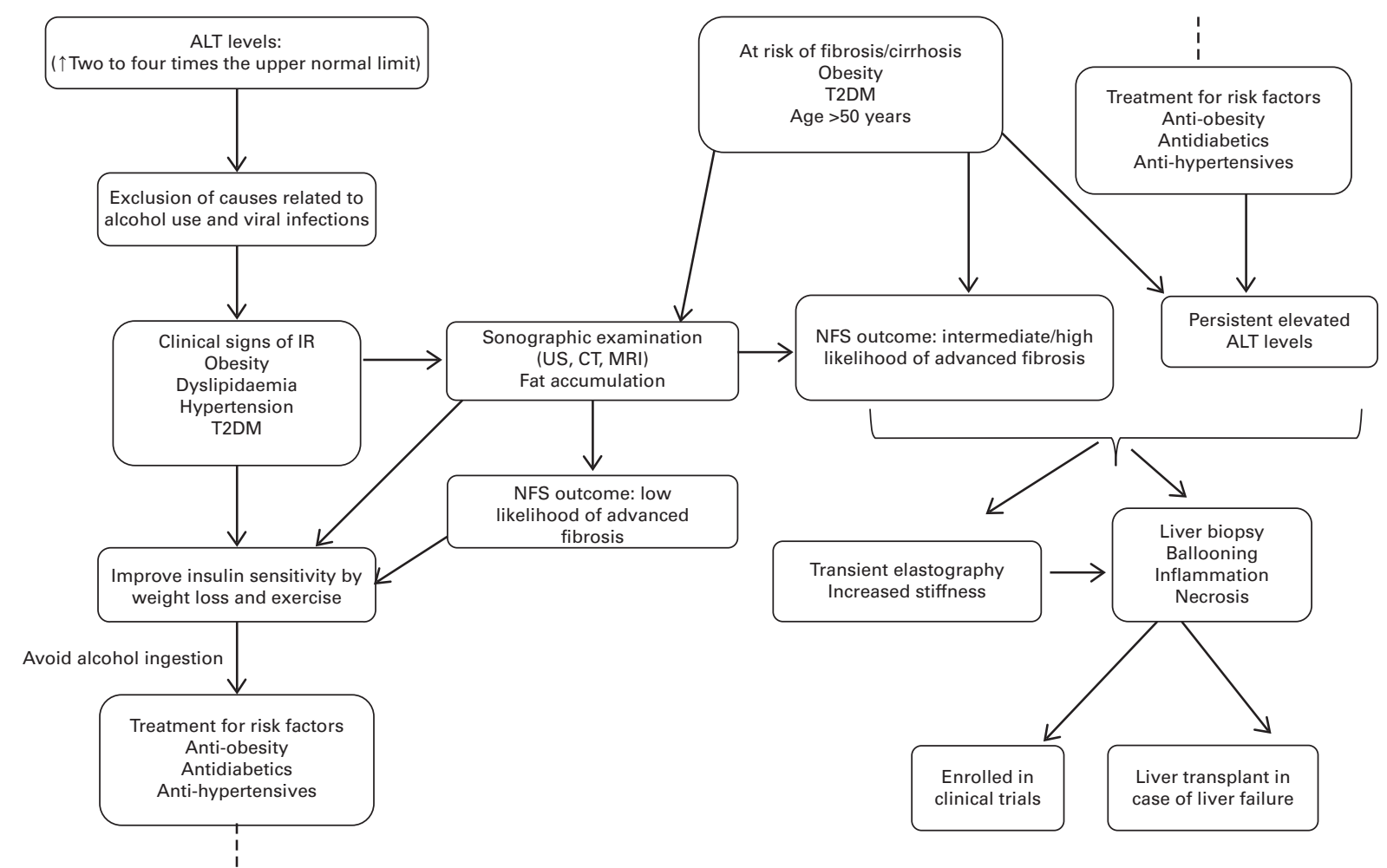

Fig. 4. Brief clinical pathway. Patients identified with increased alanine aminotransferase (ALT) levels are examined for clinical signs of insulin resistance (IR). Patients in whom IR or presence of fat accumulation is detected using ultrasonography (US) are subjected to primary weight management and methods to improve insulin sensitivity. Patients are then treated for major risk factors including diabetes, obesity and hypertension. These patients are kept under observation and periodic testing of the risk factors is carried out. If they show persistent elevated levels of ALT, then they undergo treatment similar to at-risk patients with fibrosis/cirrhosis. Following US, based on the non-alcoholic fatty liver disease fibrosis score (NFS) and ALT levels, patients are subjected to transient elastography or liver biopsy. Depending on the histopathological score of the biopsy tissue, patients are either enrolled in clinical trials or registered for a liver transplant. CT, computer tomography; IR, insulin resistance; T2DM, type 2 diabetes mellitus (note: this is not a standard care algorithm).

patients with NASH and normal ALT levels. Also, it has been shown that the levels of liver enzymes can fluctuate over the course of the disease and may be normal in end-stage cirrhosis ${ }^{(33)}$. Elderly patients ( $\geq 65$ years) are more likely to have a higher prevalence of NASH and advanced fibrosis than the non-elderly (18-64 years); therefore, liver biopsy is advised in elderly patients ${ }^{(34)}$. Among the non-invasive serum markers, the NAFLD fibrosis score is currently the most validated marker, which is widely used to differentiate the progression of NAFLD to fibrosis. A recent review by Castera et $a l .{ }^{(35)}$ has detailed the advantages and limitations of non-invasive methods for the management of NAFLD, including the diagnosis, quantification and staging of the disease. The most accurate diagnostic method is the histopathological analysis of the liver biopsy tissue. Unfortunately, this is an invasive method that is carried out only in dire conditions. The pros and cons of each of these techniques have been briefed in a review by Lomonaco et al. $^{(36)}$. Apart from these techniques, recent advances in imaging including magnetic resonance spectroscopy, liver fat measurements by proton microscopy (MRI-estimated proton density fat fraction) ${ }^{(37,38)}$, magnetic resonance elastography ${ }^{(39)}$, transient elastography (FibroScan) ${ }^{(40)}$ and estimation of plasma biomarkers such as TNF $\alpha$ and adiponectin are also being evaluated.

\section{Lean $v$. obese non-alcoholic fatty liver disease}

Although NAFLD is strongly associated with obesity, IR and type 2 diabetes, reports have suggested that it can occur even in the absence of these factors. This new entity is referred to as 'lean-NAFLD', i.e. patients with NAFLD but without obesity $\left(\mathrm{BMI}<30 \mathrm{~kg} / \mathrm{m}^{2}\right)$ and diabetes. Although the presence of the characteristics of multiple metabolic disorders is associated with a potentially progressive severe liver disease, $22 \%$ of non-diabetic patients with NAFLD had a normal $\mathrm{BMI}^{(41)}$. Liver biopsy studies in patients with chronic liver disease showed that lean-NAFLD was not only present in $2.8 \%$ of the patients, but also the most frequent cause for cryptogenic liver disease in $38 \%$ of the patients ${ }^{(42)}$. Data from the DIONYSOS study showed that the prevalence of NAFLD in the lean, non-drinking population in Italy is about $16 \%{ }^{(43)}$. Such a trend is also observed in developing countries such as India. Among the subjects from a rural population with a low prevalence of NAFLD, $75 \%$ of the patients with NAFLD had a BMI $<25 \mathrm{~kg} / \mathrm{m}^{2}$, and $54 \%$ were neither overweight nor had abdominal obesity ${ }^{(44)}$. A study conducted by Margariti et al. ${ }^{(45)}$ focused on the characteristics of NAFLD in those with a normal BMI. Their study revealed that one in every eight patients with NAFLD coming to the tertiary care had a normal BMI. When compared with overweight or obese individuals, lean patients did not show any major 
metabolic disturbances but had higher levels of AST/ALT ${ }^{(45)}$. On liver biopsy, patients with a normal BMI often exhibited NASH and histological liver lesions of similar severity to their overweight or obese counterparts. Also, the prevalence of NASH and the severity of inflammation and fibrosis did not significantly differ between patients with a normal BMI and those with increased $\mathrm{BMI}^{(46)}$. One of the reasons for ectopic fat deposition could be a slow increase in body weight $(2-10 \mathrm{~kg})$ during the adult life due to increase in adiposity ${ }^{(47)}$. These metabolically healthy but obese individuals could be at increased risk of metabolic disturbances, IR/diabetes mellitus, cardiovascular events and liver disease progression ${ }^{(48)}$. A validation study found that lean Indian men with NAFLD carried two gain-of-function SNP within the gene encoding apo3 (APOC3) ${ }^{(49)}$. However, they also had a marked IR.

These lines of evidence suggest a new direction to deal with NAFLD in lean and obese patients, such as whether the disease manifests different risk factors and progression in the presence and absence of excess weight. It is also possible that ectopic fat in lean individuals could follow a different pathophysiology ${ }^{(50)}$. One of the widely studied animal models for fatty liver is the methionine- and choline-deficient diet model, wherein the animals show decreased levels of $\beta$-oxidation and VLDL and, in turn, accumulation of fatty acids in the liver. However, these mouse models did not become obese or insulin resistant ${ }^{(51)}$. The genetically modified acylCoA:diacylglycerol acyltransferase 2 mice, which overexpress the enzyme that catalyses the final step in the biosynthesis of TAG, have been shown to develop hepatic steatosis without developing IR or impaired glucose metabolism ${ }^{(52)}$. In human subjects, SNP within patatin-like phospholipase domain-containing protein 3 have been associated with hepatic steatosis, inflammation ${ }^{(53)}$ and fibrosis ${ }^{(54)}$, but not with $\mathrm{IR}^{(55)}$. These lines of evidence and recent reports suggest a collateral investigation for unknown risk factors and secondary causes of NAFLD.

\section{Unbalanced and overnutrition - watch what you eat!}

An unbalanced nutrition has been strongly associated with NAFLD. A continued imbalance between energy expenditure and overnutrition is thought to increase adipose tissue and result in $\mathrm{IR}^{(8)}$. IR regarded as a key component of NAFLD along with increased adipose tissue favours high rates of free NEFA flux to the liver ${ }^{(56,57)}$. This adds to the TAG content in the liver, which is converted to TAG metabolites. The lipotoxic environment created by TAG metabolites is a burden on the liver, as it cannot effectively dispose the lipids either through export or by $\beta$-oxidation. Adding to this load is the continuous supply of TAG by the daily intake of foods rich in TAG-producing fuels. The decreased levels of adiponectin and high circulating levels of NEFA from muscle add to the burden on the liver (Fig. 5).

Several studies have shown that it is important to have a well-balanced combination of essential nutrients. The study of Musso et al. ${ }^{(58)}$ analysing the dietary intake of patients with NASH found that the dietary content was rich in saturated fat and cholesterol but poor in polyunsaturated fat, fibre and antioxidant vitamins $\mathrm{C}$ and E. Postprandial ApoB48 and ApoB100 responses in patients with NASH were flat and strikingly dissociated from the TAG response, suggesting a defect in ApoB secretion ${ }^{(58)}$. Also, changes in dietary fat content can change liver fat within 2 weeks, which was paralleled by changes in fasting serum insulin concentrations ${ }^{(59)}$. Apart from an imbalance in fat, higher odds of inflammation $^{(60)}$ were accompanied with higher carbohydrate

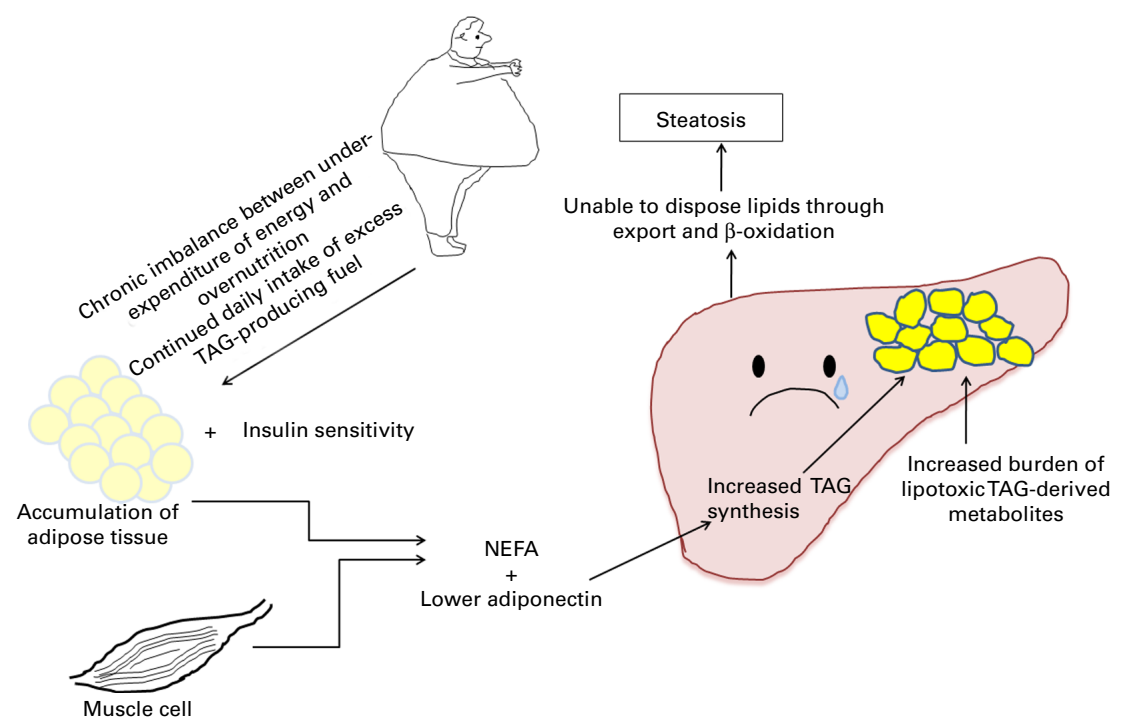

Fig. 5. Accumulation of NEFA leading to cirrhosis. A sedentary lifestyle resulting in a chronic imbalance between overnutrition and under-expenditure of energy leads to increased accumulation of adipose tissue. This is associated with insulin resistance. The accumulation of high fat mass leads to increased circulating levels of NEFA from the adipose tissue to the liver. Decreased insulin sensitivity and lower adiponectin levels further enhance the accumulation of TAG in the liver. The cycle of the daily intake of excess TAG-producing food as well as high circulating levels of NEFA from muscle and adipose tissue hampers the ability of the liver to export TAG or use it up through $\beta$-oxidation. Accumulated TAG when metabolised produce lipotoxic substances and their accumulation results in steatosis. 
intake $^{(61)}$ in NASH patients. Patients with NAFLD have a higher intake of soft drinks and meat and a tendency towards a lower intake of fish rich in $n-3$ fatty acids. Moreover, a higher intake of soft drinks and meat is associated with an increased risk of NAFLD, independent of age, sex, BMI and total energy $^{(62)}$. Increased consumption of dietary fructose (consumed in the form of high-fructose maize syrup primarily through soft drinks) has been implicated in the pathogenesis of both the MetS and NAFLD. High consumption of fructose has been postulated as a proximate cause of hepatic damage in NAFLD, and this damage is similar to the effects of ethanol or alcohol consumption ${ }^{(63)}$. Mice with ad libitum access to fructose solution showed significantly higher levels of hepatic lipid accumulation, lipid peroxidation and endotoxin levels in the portal blood as well as TNF $\alpha$ compared with controls and those fed with glucose solution ${ }^{(64)}$. Furthermore, preclinical studies in Toll-like receptor 4-mutant $(\mathrm{C} 3 \mathrm{H} / \mathrm{HeJ})$ mice showed that fructose-induced NAFLD is associated with intestinal bacterial overgrowth and increased intestinal permeability, subsequently leading to an endotoxin-dependent activation of hepatic Kupffer cells ${ }^{(65)}$. Clinical studies have shown that increased fructose consumption leads to increased fibrosis in patients with NAFLD ${ }^{(66)}$. Chronic consumption of fructose leads to an increase in serum uric acid, which poses an increased risk of hepatic ATP depletion ${ }^{(67)}$. A study conducted by Cortez-Pinto et al. ${ }^{(68)}$ in forty-five patients with NASH suggested that a combination of carbohydrate and fat intake may be more relevant than their isolated intake; increased fat intake with an excessive amount of $n$ - 6 fatty acids can be implicated in promoting necro-inflammation, and provides further grounds for individualised dietary therapy. An overview of epidemiological evidence pertaining to nutrition and PA has been reviewed in detail by Zelber-Sagi et $a{ }^{(20)}$.

\section{Treatment - non-alcoholic fatty liver disease and} non-alcoholic steatohepatitis - lifestyle modification and pharmacotherapy ${ }^{(10)}$

So far, there has been no established therapy or evidencebased clinical guidelines for NAFLD. Development of drugs for the treatment of NAFLD is currently clouded by a few issues, foremost among them being the lack of well-defined and validated end points. This is followed by long and asymptomatic natural history of the disease. In addition to these features of the disease, undefined varying phenotypes, small population studies and the lack of data to support the 'reasonably likely to predict clinical benefit' standard of surrogate points ${ }^{(69)}$ make it more difficult to treat the disease. Pharmacological treatment of patients with NAFLD is still uncertain regarding its efficacy and safety. There is no approved drug treatment for NAFLD, and the standard universally accepted as a first step is lifestyle modification. The usual management of NAFLD includes gradual weight reduction and increase in PA. Weight reduction achieved through lifestyle intervention (using a combination of diet, exercise and behaviour modification with a goal of $7-10 \%$ weight reduction) leads to improvements in liver histology in patients with $\mathrm{NASH}^{(70)}$.
Various types of diet and exercise regimen have been followed in different trials to achieve a slow but steady weight loss and improvement in liver enzymes.

Apart from these interventions, statins (lipid-lowering agents), thiozolidinediones (insulin sensitisers), antioxidants, metformin have also been used. Various trials involving these components and their effects have been reviewed in detail by Lomonaco et al. ${ }^{(36)}$ and Musso et al. ${ }^{(71)}$ (Fig. 6).

The most extensively used therapy is weight loss, thiozolidinediones and antioxidants. Weight loss is safe and improves the histology of the disease. However, a consistent challenge is faced in achieving the target weight loss. Thiozolidinediones improves steatosis and inflammation, while on the negative side, it leads to a significant weight gain. Although pioglitazone is recommended for patients with biopsy-proven $\mathrm{NASH}$, the efficacy and safety of the drug in the long-term demands more studies ${ }^{(72)}$. Furthermore, the long-term safety of using thiozolidinediones is still unclear. Randomised controlled trials with antioxidants have not always shown the same results. The effects of antioxidants vary depending on many influential factors such as age, dosage and lifestyle modifications ${ }^{(71)}$.

A study conducted in Lucknow, India with sixty-five patients showed that moderate-intensity aerobic exercise for $30 \mathrm{~min}$ daily helps in normalising ALT levels in patients with $\mathrm{NASH}^{(73)}$. An improved biochemical response was observed when lifestyle modifications (moderate sustained exercise such as brisk walking, jogging or swimming with a low-fat, low-energy diet) gave slow weight reduction, and ursodeoxycholic acid (300 mg twice per d for 6 months) was administered to patients with NAFLD showing increased levels of liver enzymes ${ }^{(74)}$. A retrospective study by Madan et $a l .{ }^{(75)}$ found that a combination of lifestyle modifications, ursodeoxycholic acid and vitamin E provided better effects than when lifestyle modifications alone was used in normalising the ALT levels. A few studies have also used metformin, which, by and large, showed normalisation of ALT levels ${ }^{(74,76)}$ even when lifestyle modifications did not have much of an effect ${ }^{(77)}$. Improved IR and histology along with a better biochemical response has been reported in twenty patients with NASH following pentoxifylline treatment for 6 months $^{(78,79)}$ (Fig. 7).

Overall, current drug therapy includes insulin sensitiser therapy, lipid-lowering drugs (which decrease VLDL, thus reducing lipid mobilisation), ursodeoxycholic acid (which mobilises hepatotoxic bile acids from their pool), betaine (which increases hepatic $S$-adenosylmethionine (SAMe)) and antioxidants (vitamin $\mathrm{E}$ and $\mathrm{N}$-acetylcysteine). However, to date, no pharmacological treatment has been shown to be effective against NAFLD ${ }^{(80)}$. Recent understanding of the molecular pathways underlying the development of NAFLD and its manifestations has led to the development of therapies targeting the mechanisms. Incretin mimetics (exenatide and liraglutide), novel insulin sensitisers (resveratrol and fenretinide), AMPK activators (oltipraz), appetite suppressants, probiotics and restoration of normal gut flora, and hepatoprotective agents such as silymarin (milk thistle) or PUFA ${ }^{(36)}$ are currently being tested. 


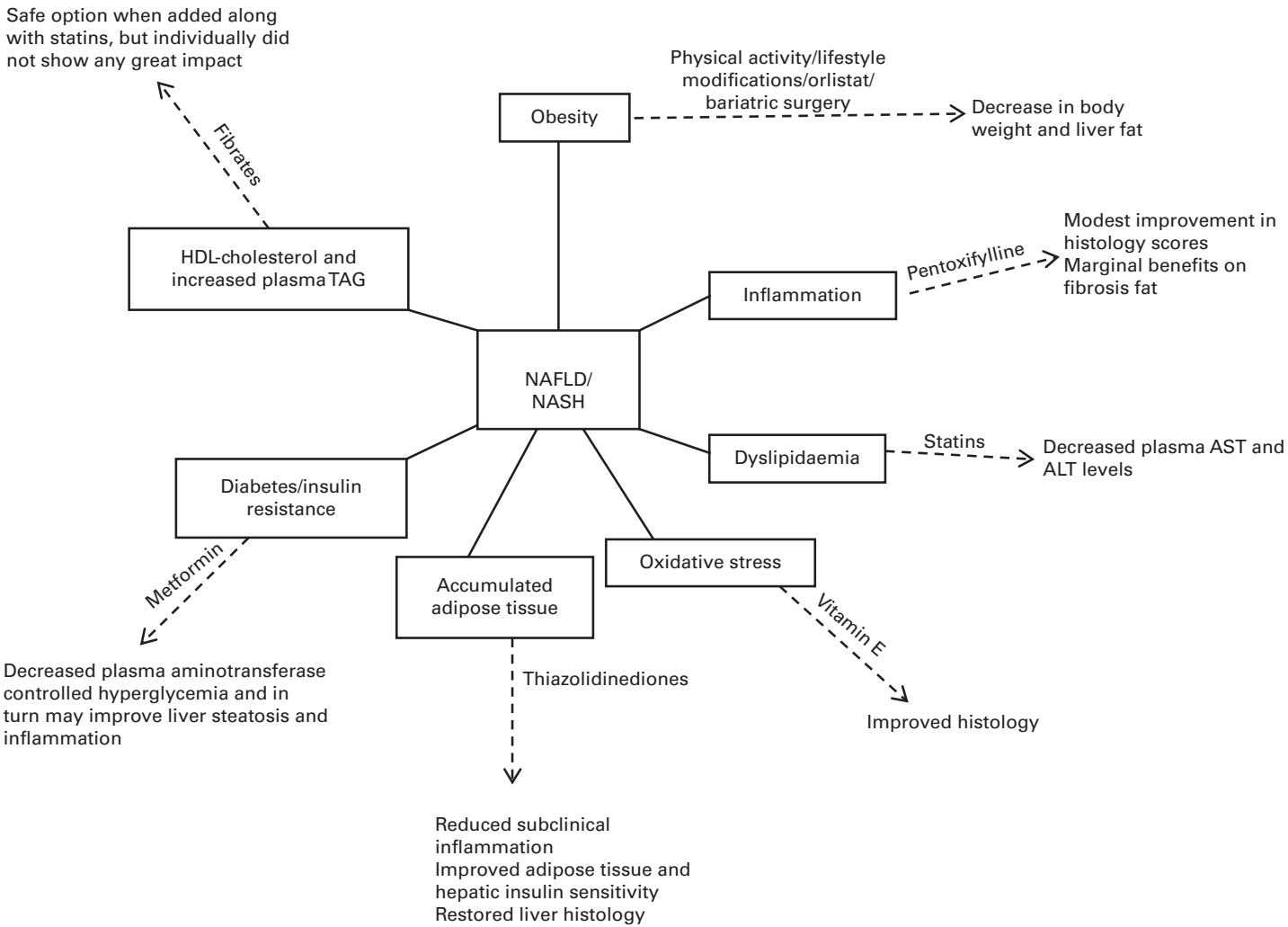

Fig. 6. Different therapeutic targets for patients with non-alcoholic fatty liver disease (NAFLD) and their broad outcomes. The data have been summarised from the studies detailed in the review by Lomonaco et al. ${ }^{(36)}$. NASH, non-alcoholic steatohepatitis; AST, aspartate aminotransferase; ALT, alanine aminotransferase.

\section{Nutrition: a promising route for prevention and management}

On the one hand, the uncertainty prevailing around the pharmacological approach to treat NAFLD with regard to its safety and efficacy and, on the other hand, weight reduction and lifestyle modifications being the first line of therapy in most cases reiterate the important role played by nutrition. An imbalance in nutrition has been shown to underlie the progression of the disease both in animals ${ }^{(81,82)}$ and in human subjects ${ }^{(59,62,68,83)}$. An unbalanced nutrition is not only strongly associated with NAFLD, but is also identified as a major risk factor that a large proportion of the population is exposed to.

The lipid content of hepatocytes is regulated by the integrated activities of cellular enzymes that catalyse lipid uptake, synthesis, oxidation and export. When 'input' of fats into these systems exceeds the capacity (either because of increased fatty acid delivery, hepatic fatty acid uptake or fatty acid synthesis) for fatty acid oxidation or export (i.e. 'output'), hepatic steatosis occurs (Fig. 5) ${ }^{(84)}$. Research in the field of nutrition and its impact on NAFLD is emerging. The major culprits identified include overconsumption of fats and carbohydrates, imbalance in macronutrients and type of dietary fat consumed (e.g. MUFA, trans-fatty acids, PUFA, cholesterol), artificially sweetened drinks and the Western dietary pattern ${ }^{(20)}$.

Lifestyle changes and weight reduction have been the widely accepted first line of management for NAFLD.
The see-saw of maintaining healthy metabolism that would help in preventing the progression of NAFLD has structured diet on the one end and PA on the other.

Over the years, the intensity of weight-reduction programmes has changed and the requirements have been pruned with better understanding of the role of different nutrients. The different regimens followed thus far has been

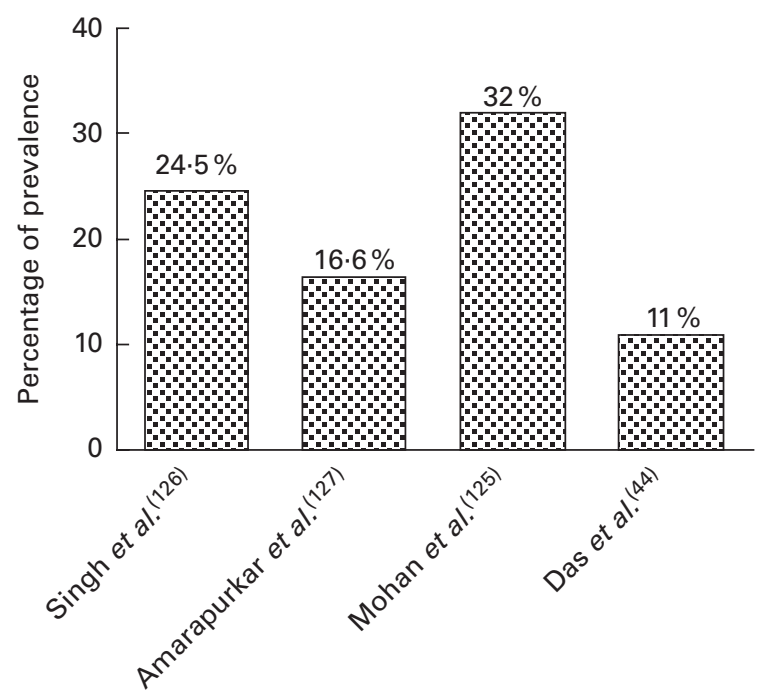

Fig. 7. Percentage of the prevalence of non-alcoholic fatty liver disease found across a few documented studies in the Indian population ${ }^{(44,125-127)}$. 
broadly classified into three pockets ${ }^{(20)}$. Although the studies have documented improvements in biochemical parameters and serum levels of enzymes, the limited number of reports with liver biopsy has been a major hindrance to strongly demonstrate the therapeutic efficacy of weight reduction. It is important to support the improvement in NAFLD condition with liver biopsy, since it is observed that certain diets may reduce liver enzymes but actually may be leading to liver damage, which is detected only through a biopsy.

The beneficial effects of PA has been supported by a number of clinical trials showing significant improvement in weight, IR, liver enzymes, TAG levels, adipose tissue volumes and levels of NEFA in plasma ${ }^{(20)}$. Apart from decreasing the symptoms of NAFLD, PA also has an overall beneficial effect on body metabolism including insulin sensitivity and glucose homeostasis ${ }^{(85)}$, NEFA metabolism ${ }^{(86)}$ and hepatic TAG content ${ }^{(87)}$, all of which are risk factors of NAFLD. The concept of resistance training over aerobic training has found significant effects in the recent past. These exercises are found to improve the lean mass and, in turn, improve insulin sensitivity ${ }^{(88)}$.

Although a large number of studies have strongly indicated the benefits of weight loss along with lifestyle modifications and PA, it has been a challenge in itself to implement these modifications in patients during the long run. Apart from PA being low in diabetic patients ${ }^{(89)}$, it has been observed that they are less likely to meet the required recommendations ${ }^{(90)}$. The situation is no different for patients diagnosed with NAFLD. In addition to very few encouraging cues towards a physically active lifestyle, fatigue has been demonstrated to be markedly higher in patients with NAFLD, which add to inactivity and excessive daytime sleepiness ${ }^{(91)}$. Although the benefits of PA and a healthy diet have been reiterated, there is still no specific regimen for PA, diet or lifestyle approach that can benefit patients with NAFLD. The need for more rigorous controlled studies for establishing evidencebased PA modifications and tailored dietary requirements has been highlighted ${ }^{(92,93)}$. In addition, weight loss is seldom maintained because low-energy diets are often discontinued; furthermore, energy restriction and weight loss are hardly feasible in non-obese subjects, who would require a different therapeutic approach ${ }^{(94)}$. Thus, there is an increased need to identify specific dietary patterns that could help to understand the pathogenesis of NAFLD/NASH, and would allow a more rationale dietary approach, before or in addition to pharmacological interventions ${ }^{(95)}$.

All these lines of evidence reiterate the difficulty in achieving success in a clinical setting and draw attention to the importance of alternative therapies for weight loss in NAFLD. The association between what you eat and NAFLD strongly conveys that balanced nutrition to prevent the progression of NAFLD is indissoluble. The identification of appropriate dietary patterns at regulated amounts can be achieved by focusing on the nutritional needs of the NAFLD condition $^{(96)}$. This can be achieved by the development of an appropriate nutrient composition delivered at measurable amounts. A slow but steady improvement in liver status along with PA and behavioural therapy in addition to rigorous follow-up would increase the chances of preventing the progression of the disease. A few of the nutrients that play an important role in maintaining liver metabolism are mentioned below (Fig. 3).

\section{Branched-chain amino acids}

Branched-chain amino acids (BCAA) are a group of essential amino acids including valine, leucine and isoleucine. A decreased serum ratio of BCAA:aromatic amino acids is regarded as a hallmark of liver cirrhosis, and normalising this ratio by supplementing BCAA through nutrition normalises the status of amino acids. Apart from albumin synthesis and decreasing the frequency of hepatocellular carcinoma development, BCAA also play an important role in glucose metabolism ${ }^{(97)}$. Studies have demonstrated that BCAA enhance glucose metabolism in the skeletal muscle, adipose tissue and liver via different molecular mechanisms. In the liver, BCAA activate $\operatorname{PPAR} \alpha$, thus increasing the oxidation of NEFA. They also decrease gluconeogenesis by increasing glucose uptake by GLUT receptors. Leucine, one of the BCAA, is known to activate the mammalian target of rapamycin/Akt pathway, which, in turn, has been found to increase the uptake of glucose and help insulin sensitivity ${ }^{(98)}$.

\section{Choline, folic acid and methionine}

Methylation plays an essential role in the normal functioning of hepatocytes. The end products of the methylation cycle result in the prevention of TAG accumulation in the liver, the production of the antioxidant glutathione and the preservation of cell membrane integrity. The main donors of methyl groups include folic acid, SAMe (principal biological methyl donor, precursor of polyamines and glutathione) and betaine (a naturally occurring metabolite of choline). The methylation of phosphatidylethanolamine to phosphotidylcholine helps in cell membrane stabilisation and VLDL synthesis that, in turn, helps in the transport of fatty acids (Fig. 3). SAMe is catalysed by methionine adenosyltransferase (MAT), specifically by MAT1A expressed mainly in the adult liver and utilised by the action of glycine $N$-methyltransferase $^{(99)}$. Studies in $M A T 1 A$-knockout mice have shown that impaired VLDL assembly mainly due to SAMe deficiency contributes to NAFLD development in these mice ${ }^{(100)}$. Similarly, glycine $N$-methyltransferase knockout mice develop liver injury, fibrosis and hepatocellular carcinoma ${ }^{(99)}$. The decrease in methylation could be either due to genetic reasons wherein the individual has decreased MAT, mutations in glycine $\mathrm{N}$-methyltransferase or no methyl donors. One of the animal models used to study the fatty liver was developed by feeding the animals with a methionine-choline-deficient diet. A number of animal studies have shown that replenishment of SAMe increased cytosolic and mitochondrial glutathione, and prevented steatosis in rats with alcohol-induced fatty liver $^{(24)}$, thus reiterating the importance of SAMe as a primary methyl donor and its important role in hepato-physiology. Diet is one of the major routes by which the levels of these methyl donors could be replenished. Betaine methylates 
homocysteine to methionine, which, in turn, can be converted to SAMe (Fig. 3) in the presence of betaine-homocysteine methyltransferase. Betaine has been shown to improve liver transaminases in patients with $\mathrm{NASH}$ in a pilot study ${ }^{(101)}$, and to protect against worsening in a subsequent study ${ }^{(102)}$. With the knowledge of enzymes and genes playing an important role as rate-limiting steps, it would be beneficial to consider their expression for a positive result in the presence of the substrate. With studies showing positive effects of methyl donors in liver disease ${ }^{(101,103)}$, it would be appropriate to incorporate these ingredients in nutritional components for the therapy of NAFLD.

\section{Vitamin $E$}

Vitamin E belongs to a class of lipid-soluble tocopherols that exhibit antioxidant activity. This property of vitamin E gives it an important role to play in lipid peroxidation and membrane stabilisation. Apart from being an antioxidant, it is also found to attenuate the cytokine-induced stimulation of stellate cells by decreasing the levels of transforming growth factor $\beta^{(104)}$, and reduce mitochondrial lipid peroxidation and, in turn, correct oxidative stress. It has also shown effective results in animal models of liver disease associated with oxidative injury ${ }^{(105,106)}$. This would decrease collagen deposition and progression to fibrosis (Fig. 3). These properties of vitamin $\mathrm{E}$ indicate that it has beneficial effects on NAFLD/NASH-like conditions, and thus have been used in multiple clinical trials ${ }^{(24)}$.

The distinction between simple steatosis and NASH can only be done by means of a liver biopsy; however, strategies that reduce fatty liver disease correlate with a reduction in steatohepatitis and fibrosis. Pharmacological therapy, at large, has been ineffective in the treatment of NAFLD/ $\mathrm{NASH}$, including trials using antioxidants and cytoprotective agents such as pentoxifylline, vitamin $\mathrm{E}$ and ursodeoxycholic acid. As awareness of NAFLD/NASH increases, health care providers are now being confronted with the unique challenge of achieving good metabolic control while treating fatty liver disease. Inadequate glycaemic control appears to worsen and accelerate the progression of fatty liver disease. Paradoxically, most patients with type 2 diabetes mellitus do not reach established treatment goals. Moreover, it appears that the way by which metabolic control is achieved is important in NAFLD: while metformin may be beneficial in NASH and pioglitazone is highly effective ${ }^{(107)}$, the control of hyperglycaemia by insulin therapy alone has modest benefits on hepatic fat accumulation ( $\mathrm{K}$ Cusi, unpublished results). In addition, long-term insulin therapy in patients with type 2 diabetes mellitus is frequently associated with inadequate compliance, weight gain, frequent hypoglycaemia and overall poor patient satisfaction. This is of great concern as approximately $35-40 \%$ of patients with type 2 diabetes mellitus are treated with insulin. Therefore, there is an unmet need to find better ways to treat patients who fail oral drug therapy, targeting both hyperglycaemia and excessive liver fat deposition.
Nutritional ingredients in liver disease: silymarin, green tea and spirulina

Silybum marianum (milk thistle) has two predominant and most active components: silymarin, a mixture of flavanoliganans and silibinin. Both these components are found to exert hepato-protective, antioxidant, anti-inflammatory and anti-fibrotic properties ${ }^{(108)}$. Silymarin extract was found to ameliorate carbon tetrachloride-induced ${ }^{(109)}$ and methionineand choline-deficient diet-induced ${ }^{(110)}$ liver fibrosis, and reduced the activation of hepatic stellate cells. Silibinin treatment in a $d b / d b$ methionine- and choline-deficient mouse model was found to counteract the progression of liver injury by modulating lipid homeostasis and suppressing oxidative stress-mediated lipotoxicity and NF- $\kappa \mathrm{B}$ activation ${ }^{(111)}$ End analysis of clinical and biochemical parameters in a randomised controlled pilot study of patients with NAFLD showed that most reduction in the average amount of AST and ALT occurred in the silymarin group ${ }^{(112)}$ when compared with the pioglitazone and metformin groups. In another study, patients with NAFLD on a food supplement containing silymarin also showed improved AST and ALT levels in their serum after 6 months ${ }^{(113)}$. Although the underlying mechanisms are not clearly understood, milk thistle seems to bring about its protective effects by acting as an antioxidant ${ }^{(114)}$. Since mitochondrial dysfunction in $\beta$-oxidation leading to the generation of reactive oxygen species and the activation of stellate cells for inflammatory reactions are the consequences of NAFLD that lead to the progression of the disease, the prevention of such a chain of reaction could be beneficial in the treatment strategy for NAFLD. Milk thistle could address the generation of reactive oxygen species and, in turn, keep the condition from worsening.

The beneficial effects of green tea extract in protecting against NAFLD seem to be due to catechins ${ }^{(115)}$ that regulate energy homeostasis (reducing dietary lipid and carbohydrate absorption, decreasing lipogenesis, increasing energy expenditure) and decrease oxidative stress, enzyme inhibition and inflammatory response. Green tea extract has been shown to protect against NAFLD by limiting hepatic lipid accumulation $^{(116)}$ and adipose lipogenesis ${ }^{(117)}$ in an $o b / o b$ mouse model and by increasing glutathione levels and decreasing NF- $\mathrm{KB}$ activity ${ }^{(118)}$ in a high-fat diet-fed rat model. Several animal models ${ }^{(119)}$ and clinical trials have addressed the positive effects of green tea consumption on the prevention of liver diseases ${ }^{(120)}$. It is primarily thought to bring about its protective effects by the antioxidant and anti-inflammatory mechanisms of catechins.

Another ingredient that has been extensively studied in liver diseases is spirulina (Arthrospira), a filamentous unicellular blue green alga. Wistar rats receiving 5\% Arthrospira maxima in their diet significantly lowered serum AST and liver TAG levels in a carbon tetrachloride-induced steatohepatitis model. Their results suggested an antioxidant mechanism and a lower unsaturation of the liver fatty acids for the protection $^{(121)}$. Spirulina feeding for 12 weeks in a monosodium glutamate-induced model of IR and NASH has shown beneficial effects on serum cholesterol and TAG levels, liver 


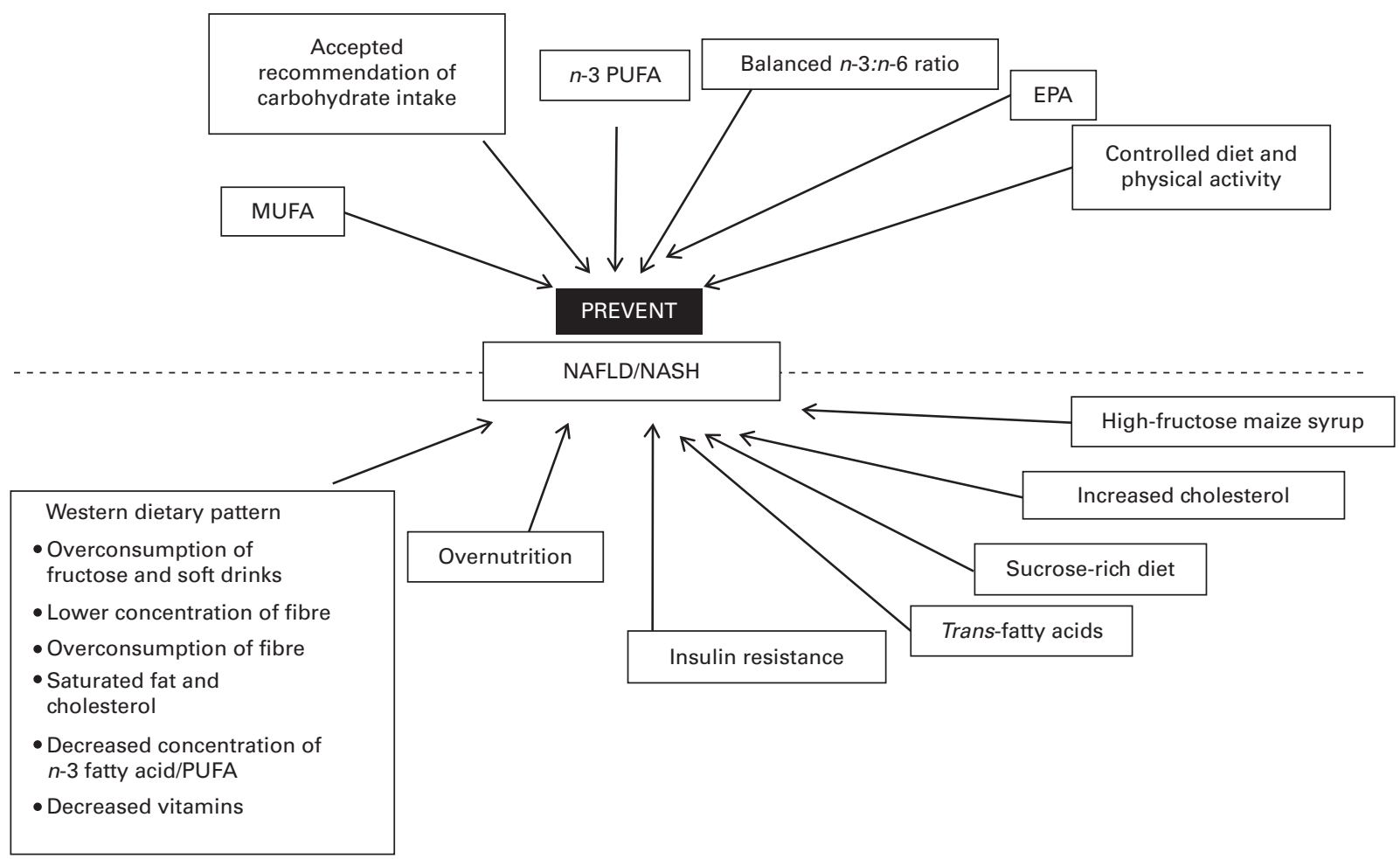

Fig. 8. Watch what you eat! With nutrition playing a vital role in non-alcoholic fatty liver disease (NAFLD), certain balanced foods have found to be effective in preventing the development of NAFLD or its progression (top half of the figure). In contrast, consumption of certain kinds of diets (bottom half of the figure) in the long run invariably disturbs the metabolism and leads to the conditions such as NAFLD. PUFA, MUFA, EPA (devised from Zelber-Sagi et al. ${ }^{(62)}$ ). NASH, nonalcoholic steatohepatitis.

NEFA levels and lowered leptin levels. Histological analysis showed decreased NAS and lipid peroxidation in spirulinafed mice. It has been speculated that spirulina prevents the progression of NAFLD by acting as an anti-inflammatory agent $^{(122)}$. At least three Hispanic Mexican patients treated with $4.5 \mathrm{~g} / \mathrm{d}$ of spirulina for 3 months showed improvement in their ultrasonography and aminotransferase data and also had hypolipidaemic effects when compared with before treatment $^{(123)}$

A study by Dunn et al. ${ }^{(124)}$ supports the safety of one glass of wine per $\mathrm{d}$ for cardioprotection in patients at risk for both CHD and NAFLD. Further detailed randomised controlled trials in individuals with NAFLD are required to examine the potentially beneficial effects of these ingredients. A thorough follow-up of patients and detailed in vivo animal experiments would further unravel the mechanisms underlying the positive effects of these ingredients in the long run. This knowledge can be safely used as a productive therapeutic strategy in conditions such as NAFLD, where the use of pharmacotherapy is still uncertain.

\section{Conclusion}

NAFLD is not a separate disease that affects the liver, but a preamble to more disorder in the metabolism when overlooked. The non-availability of proper diagnostic tools, the lack of awareness among the patients about future complications, and the condition being cloaked without symptoms in the beginning makes it more of a 'put off' problem. However, the increasing reports of fatty liver across the world have now added momentum in understanding the mechanisms of NAFLD and its progression. The lack of a targeted therapy demands a thorough understanding of the cause and effects of the disorder and development of new therapeutic strategies that can prevent its progression. With increasing reports of obesity and diabetes, the main risk factors for NAFLD, it is highly possible that a large population is affected by NAFLD. This in addition to the Western diet and sedentary lifestyle increases the probability of patients with NAFLD. Studies increasingly have suggested that dietary habits may promote steatohepatitis directly by modulating hepatic TAG accumulation and antioxidant activity, as well as indirectly by affecting insulin sensitivity and postprandial TAG metabolism ${ }^{(58)}$ (Fig. 8).

However, dietary patterns are not uniform across all patients, and it is increasingly challenging to reduce weight and maintain reduced weight in the long term. Thus, there is a need to develop more specific, alimentary interventions that can meet the requirements of not only the diabetics or obese but also the non-obese, non-diabetic, normolipidaemics who are also prone to NAFLD/NASH. Changing the dietary composition without necessarily reducing energy intake may offer a more realistic and feasible alternative to treat patients with NAFLD ${ }^{(20)}$. With no definite pharmacological treatment for NAFLD, and it being subject to the stage of the disease based on the results for biopsy, it is indeed a recommended 
step to suppress the progression of the disease at early stages using nutritional interventions. Nutritional therapy along with PA and pharmacological therapies might provide a better platform to impede the progression of the NAFLD spectrum. Such an approach can also have a control over the associated risk factors of NAFLD, which is growing at a disturbing rate across the world.

\section{Acknowledgements}

The authors' contributions are as follows: J. V. designed and drafted the review; J. V., S. Si. and S. Su. contributed to the conception of the review; J. V., A. M., S. Su. and S. Si. revised the manuscript critically for its content and arrangement; S. Si. approved the final version of the manuscript.

There are no conflicts of interest to declare.

\section{References}

1. Ludwig J, Viggiano TR, McGill DB, et al. (1980) Nonalcoholic steatohepatitis: Mayo Clinic experiences with a hitherto unnamed disease. Mayo Clin Proc 55, 434-438.

2. Dhiman RK \& Duseja A (2005) Nonalcoholic fatty liver disease. J Assoc Physicians India 15, 434-438.

3. Baker AL (1985) Nonalcoholic steatonecrosis: a unique histopathologic lesion of the liver with multiple causes. Surg Dig Dis 3, 154-164.

4. Adler M \& Schaffner F (1979) Fatty liver hepatitis and cirrhosis in obese patients. Am J Med 67, 811-816.

5. French SW, Eidus LB \& Freeman J (1989) Nonalcoholic fatty hepatitis: an important clinical condition. Can J Gastroenterol 3, 189-197.

6. Sanyal AJ (2002) AGA technical review on nonalcoholic fatty liver disease. Gastroenterology 123, 1705-1725.

7. Caldwell SH, Oelsner DH, Iezzoni JC, et al. (1999) Cryptogenic cirrhosis: clinical characterization and risk factors for underlying disease. Hepatology 29, 664-669.

8. Vos MB \& McClain CJ (2008) Nutrition and nonalcoholic fatty liver disease in children. Curr Gastroenterol Rep 10, $308-315$

9. Bellentani S, Scaglioni F, Marino M, et al. (2010) Epidemiology of non-alcoholic fatty liver disease. Dig Dis $\mathbf{2 8}$, $155-161$

10. Centis E, Marzocchi R, Suppini A, et al. (2013) The role of lifestyle change in the prevention and treatment of NAFLD. Curr Pharm Des 19, 5270-5279.

11. Festi D, Schiumerini R, Marzi L, et al. (2013) Review Article: the diagnosis of non-alcoholic fatty liver disease - availability and accuracy of non-invasive methods. Aliment Pharmacol Ther 37, 392-400.

12. Deepak A (2011) NAFLD and NASH: Asian perspective. Trop Gatroenterol 32, Suppl. 1, S23-S26.

13. WGOG Guidelines (2012) Nonalcoholic Fatty Liver Disease and Nonalcoholic Steatohepatitis, vol. 17. Milwaukee: WGOG.

14. Dowman JK, Tomlinson JW \& Newsome PN (2010) Pathogenesis of non-alcoholic fatty liver disease. $O J M$ 103, 71-83

15. Dharel N \& Fuchs M (2014) Nonalcoholic fatty liver disease - a major public health challenge for the 21st century. JSM Gastroenterol Hepatol 2, 1018.
16. Jou J, Choi SS \& Diehl AM (2008) Mechanisms of disease progression in nonalcoholic fatty liver disease. Semin Liver Dis 28, 370-379.

17. Chiang DJ, Pritchard MT \& Nagy LE (2011) Obesity, diabetes mellitus, and liver fibrosis. Am J Physiol Gastrointest Liver Physiol 300, G697-G702.

18. Malaguarnera M, Di Rosa M, Nicoletti F, et al. (2009) Molecular mechanisms involved in NAFLD progression. J Mol Med (Berl) 87, 679-695.

19. Duseja A (2010) Nonalcoholic fatty liver disease in India - a lot done, yet more required! Indian J Gastroenterol 29, $217-225$.

20. Zelber-Sagi S, Ratziu V \& Oren R (2011) Nutrition and physical activity in NAFLD: an overview of the epidemiological evidence. World J Gastroenterol 17, 3377-3389.

21. Kim CH \& Younossi ZM (2008) Nonalcoholic fatty liver disease: a manifestation of the metabolic syndrome. Cleve Clin J Med 75, 721-728.

22. Tarantino G \& Finelli C (2013) What about non-alcoholic fatty liver disease as a new criterion to define metabolic syndrome? World J Gastroenterol 19, 3375-3384.

23. Day CP \& James OF (1998) Steatohepatitis: a tale of two "hits"? Gastroenterology 114, 842-845.

24. Chang CY, Argo CK, Al-Osaimi AM, et al. (2006) Therapy of NAFLD: antioxidants and cytoprotective agents. I Clin Gastroenterol 40, Suppl. 1, S51-S60

25. Polyzos SA, Kountouras J, Zavos C, et al. (2010) The role of adiponectin in the pathogenesis and treatment of non-alcoholic fatty liver disease. Diabetes Obes Metab 12, 365-383.

26. Finelli C \& Tarantino G (2013) What is the role of adiponectin in obesity related non-alcoholic fatty liver disease? World J Gastroenterol 19, 802-812.

27. Chalasani N, Younossi Z, Lavine JE, et al. (2012) The diagnosis and management of non-alcoholic fatty liver disease practice guideline by the American Gastroenterological Association, American Association for the Study of Liver Diseases, and American College of Gastroenterology. Gastroenterology 142, 1592-1609.

28. Ratziu V, Bellentani S, Cortez-Pinto H, et al. (2010) A position statement on NAFLD/NASH based on the EASL 2009 special conference. J Hepatol 53, 372-384.

29. Tarantino G, Scopacasa F, Colao A, et al. (2011) Serum Bcl-2 concentrations in overweight-obese subjects with nonalcoholic fatty liver disease. World J Gastroenterol 17, $5280-5288$

30. Moylan CA, Pang H, Dellinger A, et al. (2014) Hepatic gene expression profiles differentiate presymptomatic patients with mild versus severe nonalcoholic fatty liver disease. Hepatology 59, 471-482.

31. Burgert TS, Taksali SE, Dziura J, et al. (2006) Alanine aminotransferase levels and fatty liver in childhood obesity: associations with insulin resistance, adiponectin, and visceral fat. I Clin Endocrinol Metab 91, 4287-4294.

32. Amarapurkar DN \& Patel ND (2004) Clinical spectrum and natural history of non-alcoholic steatohepatitis with normal alanine aminotransferase values. Trop Gastroenterol 25, $130-134$.

33. Ipekci SH, Basaranoglu M \& Sonsuz A (2003) The fluctuation of serum levels of aminotransferase in patients with nonalcoholic steatohepatitis. J Clin Gastroenterol 36, 371

34. Noureddin M, Yates KP, Vaughn IA, et al. (2013) Clinical and histological determinants of nonalcoholic steatohepatitis and advanced fibrosis in elderly patients. Hepatology $\mathbf{5 8}$, $1644-1654$ 
35. Castera L, Vilgrain V \& Angulo P (2013) Noninvasive evaluation of NAFLD. Nat Rev Gastroenterol Hepatol 10 , 666-675.

36. Lomonaco R, Sunny NE, Bril F, et al. (2013) Nonalcoholic fatty liver disease: current issues and novel treatment approaches. Drugs $\mathbf{7 3}, 1-14$.

37. Tang A, Tan J, Sun M, et al. (2013) Nonalcoholic fatty liver disease: MR imaging of liver proton density fat fraction to assess hepatic steatosis. Radiology 267, 422-431.

38. Reeder SB, Hu HH \& Sirlin CB (2012) Proton density fatfraction: a standardized MR-based biomarker of tissue fat concentration. J Magn Reson Imaging 36, 1011-1014.

39. Mariappan YK, Glaser KJ \& Ehman RL (2010) Magnetic resonance elastography: a review. Clin Anat 23, 497-511.

40. Foucher J, Chanteloup E, Vergniol J, et al. (2006) Diagnosis of cirrhosis by transient elastography (FibroScan): a prospective study. Gut 55, 403-408.

41. Marchesini G, Bugianesi E, Forlani G, et al. (2003) Nonalcoholic fatty liver, steatohepatitis, and the metabolic syndrome. Hepatology 37, 917-923.

42. Vos B, Moreno C, Nagy N, et al. (2011) Lean non-alcoholic fatty liver disease (lean-NAFLD): a major cause of cryptogenic liver disease. Acta Gastroenterol Belg 74, 389-394.

43. Bellentani S, Saccoccio G, Masutti F, et al. (2000) Prevalence of and risk factors for hepatic steatosis in Northern Italy. Ann Intern Med 132, 112-117.

44. Das K, Mukherjee PS, Ghosh A, et al. (2010) Nonobese population in a developing country has a high prevalence of nonalcoholic fatty liver and significant liver disease. Hepatology 51, 1593-1602.

45. Margariti E, Deutsch M \& Manolakopoulos S (2012) Nonalcoholic fatty liver disease may develop in individuals with normal body mass index. Ann Gastroenterol $\mathbf{2 5}$, $45-51$.

46. Margariti A, Deutsch M, Manolakopoulos S, et al. (2013) The severity of histologic liver lesions is independent of body mass index in patients with nonalcoholic fatty liver disease. J Clin Gastroenterol 47, 280-286.

47. Ruderman N, Chisholm D, Pi-Sunyer X, et al. (1998) The metabolically obese, normal-weight individual revisited. Diabetes 47, 699-713.

48. Messier V, Karelis AD, Robillard ME, et al. (2010) Metabolically healthy but obese individuals: relationship with hepatic enzymes. Metabolism 59, 20-24.

49. Petersen KF, Dufour S, Hariri A, et al. (2010) Apolipoprotein $\mathrm{C} 3$ gene variants in nonalcoholic fatty liver disease. $N \mathrm{Engl}$ J Med 362, 1082-1089

50. Verdelho Machado M \& Cortez-Pinto H (2012) Fatty liver in lean patients: is it a different disease? Ann Gastroenterol 25, $1-2$.

51. Anstee QM (2011) Animal models in nonalcoholic steatohepatitis research: utility and clinical translation. Liver Int 31, 440-442.

52. Monetti M, Levin MC, Watt MJ, et al. (2007) Dissociation of hepatic steatosis and insulin resistance in mice overexpressing DGAT in the liver. Cell Metab 6, 69-78.

53. Rotman Y, Koh C, Zmuda JM, et al. (2010) The association of genetic variability in patatin-like phospholipase domaincontaining protein 3 (PNPLA3) with histological severity of nonalcoholic fatty liver disease. Hepatology 52, 894-903.

54. Valenti L, Al-Serri A, Daly AK, et al. (2010) Homozygosity for the patatin-like phospholipase-3/adiponutrin I148M polymorphism influences liver fibrosis in patients with nonalcoholic fatty liver disease. Hepatology 51, 1209-1217.

55. Romeo S, Huang-Doran I, Baroni MG, et al. (2010) Unravelling the pathogenesis of fatty liver disease: patatin-like phospholipase domain-containing 3 protein. Curr Opin Lipidol 21, 247-252.

56. Cusi K (2012) Role of obesity and lipotoxicity in the development of nonalcoholic steatohepatitis: pathophysiology and clinical implications. Gastroenterology 142, $711-725 . e 716$.

57. Fabbrini E, Mohammed BS, Magkos F, et al. (2008) Alterations in adipose tissue and hepatic lipid kinetics in obese men and women with nonalcoholic fatty liver disease. Gastroenterology 134, 424-431.

58. Musso G, Gambino R, De Michieli F, et al. (2003) Dietary habits and their relations to insulin resistance and postprandial lipemia in nonalcoholic steatohepatitis. Hepatology 37, 909-916.

59. Westerbacka J, Lammi K, Hakkinen AM, et al. (2005) Dietary fat content modifies liver fat in overweight nondiabetic subjects. J Clin Endocrinol Metab 90, 2804-2809.

60. Solga S, Alkhuraishe AR, Clark JM, et al. (2004) Dietary composition and nonalcoholic fatty liver disease. Dig Dis Sci 49, 1578-1583.

61. Toshimitsu K, Matsuura B, Ohkubo I, et al. (2007) Dietary habits and nutrient intake in non-alcoholic steatohepatitis. Nutrition 23, 46-52.

62. Zelber-Sagi S, Nitzan-Kaluski D, Goldsmith R, et al. (2007) Long term nutritional intake and the risk for non-alcoholic fatty liver disease (NAFLD): a population based study. J Hepatol 47, 711-717.

63. Lim JS, Mietus-Snyder M, Valente A, et al. (2010) The role of fructose in the pathogenesis of NAFLD and the metabolic syndrome. Nat Rev Gastroenterol Hepatol 7, 251-264.

64. Bergheim I, Weber S, Vos M, et al. (2008) Antibiotics protect against fructose-induced hepatic lipid accumulation in mice: role of endotoxin. J Hepatol 48, 983-992.

65. Spruss A, Kanuri G, Wagnerberger S, et al. (2009) Tolllike receptor 4 is involved in the development of fructose-induced hepatic steatosis in mice. Hepatology 50, 1094-1104.

66. Abdelmalek MF, Suzuki A, Guy C, et al. (2010) Increased fructose consumption is associated with fibrosis severity in patients with nonalcoholic fatty liver disease. Hepatology 51, 1961-1971.

67. Abdelmalek MF, Lazo M, Horska A, et al. (2012) Higher dietary fructose is associated with impaired hepatic adenosine triphosphate homeostasis in obese individuals with type 2 diabetes. Hepatology 56, 952-960.

68. Cortez-Pinto H, Jesus L, Barros H, et al. (2006) How different is the dietary pattern in non-alcoholic steatohepatitis patients? Clin Nutr 25, 816-823.

69. Dimick-Santos L (2013) FDA perspectives: key needs in the field to guide drug development in NASH and fibrosis. In Trial Designs and Endpoints for Liver Disease Secondary to Nonalcoholic Fatty Liver Disease (NAFLD), pp. 7-11 [J Arun, AJM Sanyal, SL Friedman and L Dimick-Santos, editors]. FDA: White Oak Campus, Silver Spring, MD

70. Promrat K, Kleiner DE, Niemeier HM, et al. (2010) Randomized controlled trial testing the effects of weight loss on nonalcoholic steatohepatitis. Hepatology 51, 121-129.

71. Musso G, Gambino R, Cassader M, et al. (2010) A meta-analysis of randomized trials for the treatment of nonalcoholic fatty liver disease. Hepatology 52, 79-104.

72. Chalasani N, Younossi Z, Lavine JE, et al. (2012) The diagnosis and management of non-alcoholic fatty liver disease: practice guideline by the American Association for the Study of Liver Diseases, American College of Gastroenterology, and the American Gastroenterological Association. Hepatology 55, 2005-2023. 
73. Sreenivasa Baba C, Alexander G, Kalyani B, et al. (2006) Effect of exercise and dietary modification on serum aminotransferase levels in patients with nonalcoholic steatohepatitis. J Gastroenterol Hepatol 21, 191-198.

74. Duseja A, Das A, Das R, et al. (2007) The clinicopathological profile of Indian patients with nonalcoholic fatty liver disease (NAFLD) is different from that in the West. Dig Dis Sci 52, 2368-2374.

75. Madan K, Batra Y, Gupta DS, et al. (2005) Vitamin E-based therapy is effective in ameliorating transaminasemia in nonalcoholic fatty liver disease. Indian J Gastroenterol $\mathbf{2 4}$ $251-255$

76. Duseja A, Murlidharan R, Bhansali A, et al. (2004) Assessment of insulin resistance and effect of metformin in nonalcoholic steatohepatitis - a preliminary report. Indian J Gastroenterol 23, 12-15.

77. Duseja A, Das A, Dhiman RK, et al. (2007) Metformin is effective in achieving biochemical response in patients with nonalcoholic fatty liver disease (NAFLD) not responding to lifestyle interventions. Ann Hepatol 6, 222-226.

78. Satapathy SK, Garg S, Chauhan R, et al. (2004) Beneficial effects of tumor necrosis factor-alpha inhibition by pentoxifylline on clinical, biochemical, and metabolic parameters of patients with nonalcoholic steatohepatitis. Am J Gastroenterol 99, 1946-1952.

79. Satapathy SK, Sakhuja P, Malhotra V, et al. (2007) Beneficial effects of pentoxifylline on hepatic steatosis, fibrosis and necroinflammation in patients with non-alcoholic steatohepatitis. J Gastroenterol Hepatol 22, 634-638.

80. Lam BP \& Younossi ZM (2009) Treatment regimens for non-alcoholic fatty liver disease. Ann Hepatol 8, Suppl. 1, S51-S59

81. Poulsom R (1986) Morphological changes of organs after sucrose or fructose feeding. Prog Biochem Pharmacol 21 , 104-134.

82. Bogin E, Avidar Y \& Merom M (1986) Biochemical changes in liver and blood during liver fattening in rats. J Clin Chem Clin Biochem 24, 621-626.

83. Assy N, Nasser G, Kamayse I, et al. (2008) Soft drink consumption linked with fatty liver in the absence of traditional risk factors. Can J Gastroenterol 22, 811-816.

84. Koteish A \& Diehl AM (2001) Animal models of steatosis. Semin Liver Dis 21, 89-104.

85. Boule NG, Haddad E, Kenny GP, et al. (2001) Effects of exercise on glycemic control and body mass in type 2 diabetes mellitus: a meta-analysis of controlled clinical trials. JAMA 286, 1218-1227.

86. Hannukainen JC, Nuutila P, Borra R, et al. (2007) Increased physical activity decreases hepatic free fatty acid uptake: a study in human monozygotic twins. J Physiol 578, 347-358.

87. Tamura Y, Tanaka Y, Sato F, et al. (2005) Effects of diet and exercise on muscle and liver intracellular lipid contents and insulin sensitivity in type 2 diabetic patients. J Clin Endocrinol Metab 90, 3191-3196.

88. Ballor DL \& Keesey RE (1991) A meta-analysis of the factors affecting exercise-induced changes in body mass, fat mass and fat-free mass in males and females. Int $J$ Obes 15, 717-726.

89. Morrato EH, Hill JO, Wyatt HR, et al. (2007) Physical activity in U.S. adults with diabetes and at risk for developing diabetes, 2003. Diabetes Care 30, 203-209.

90. Zhao G, Ford ES, Li C, et al. (2008) Compliance with physical activity recommendations in US adults with diabetes. Diabet Med 25, 221-227.

91. Newton JL, Jones DE, Henderson E, et al. (2008) Fatigue in non-alcoholic fatty liver disease (NAFLD) is significant and associates with inactivity and excessive daytime sleepiness but not with liver disease severity or insulin resistance. Gut 57, 807-813.

92. Finelli C \& Tarantino G (2012) Have guidelines addressing physical activity been established in nonalcoholic fatty liver disease? World J Gastroenterol 18, 6790-6800.

93. Finelli C \& Tarantino G (2012) Is there any consensus as to what diet or lifestyle approach is the right one for NAFLD patients? J Gastrointestin Liver Dis 21, 293-302.

94. Angulo P \& Lindor KD (2001) Treatment of nonalcoholic fatty liver: present and emerging therapies. Semin Liver Dis 21, 81-88.

95. Fan JG \& Cao HX (2013) Role of diet and nutritional management in non-alcoholic fatty liver disease. $J$ Gastroenterol Hepatol 28, Suppl. 4, 81-87.

96. McCarthy EM \& Rinella ME (2012) The role of diet and nutrient composition in nonalcoholic fatty liver disease. J Acad Nutr Diet 112, 401-409.

97. Layman DK, Shiue H, Sather C, et al. (2003) Increased dietary protein modifies glucose and insulin homeostasis in adult women during weight loss. J Nutr 133, 405-410.

98. Kawaguchi T, Izumi N, Charlton MR, et al. (2011) Branched-chain amino acids as pharmacological nutrients in chronic liver disease. Hepatology 54, 1063-1070.

99. Lu SC \& Mato JM (2012) S-Adenosylmethionine in liver health, injury, and cancer. Physiol Rev 92, 1515-1542.

100. Cano A, Buqué X, Martínez-Uña M, et al. (2011) Methionine adenosyltransferase 1A gene deletion disrupts hepatic very low-density lipoprotein assembly in mice. Hepatology $\mathbf{5 4}$ 1975-1986.

101. Abdelmalek MF, Angulo P, Jorgensen RA, et al. (2001) Betaine, a promising new agent for patients with nonalcoholic steatohepatitis: results of a pilot study. $A m J$ Gastroenterol 96, 2711-2717.

102. Abdelmalek MF, Sanderson SO, Angulo P, et al. (2009) Betaine for nonalcoholic fatty liver disease: results of a randomized placebo-controlled trial. Hepatology 50, $1818-1826$

103. Vendemiale G, Altomare E, Trizio T, et al. (1989) Effects of oral $S$-adenosyl-L-methionine on hepatic glutathione in patients with liver disease. Scand J Gastroenterol 24, 407-415.

104. Parola M, Muraca R, Dianzani I, et al. (1992) Vitamin E dietary supplementation inhibits transforming growth factor beta 1 gene expression in the rat liver. FEBS Lett 308, 267-270.

105. Soltys K, Dikdan G \& Koneru B (2001) Oxidative stress in fatty livers of obese Zucker rats: rapid amelioration and improved tolerance to warm ischemia with tocopherol Hepatology 34, 13-18.

106. Parola M, Leonarduzzi G, Biasi F, et al. (1992) Vitamin E dietary supplementation protects against carbon tetrachloride-induced chronic liver damage and cirrhosis Hepatology 16, 1014-1021.

107. Belfort R, Harrison SA, Brown K, et al. (2006) A placebo-controlled trial of pioglitazone in subjects with nonalcoholic steatohepatitis. $N$ Engl J Med 355, 2297-2307.

108. Pradhan SC \& Girish C (2006) Hepatoprotective herbal drug, silymarin from experimental pharmacology to clinical medicine. Indian J Med Res 124, 491-504.

109. Tsai JH, Liu JY, Wu TT, et al. (2008) Effects of silymarin on the resolution of liver fibrosis induced by carbon tetrachloride in rats. J Viral Hepat 15, 508-514.

110. Kim M, Yang SG, Kim JM, et al. (2012) Silymarin suppresses hepatic stellate cell activation in a dietary rat model of 
non-alcoholic steatohepatitis: analysis of isolated hepatic stellate cells. Int J Mol Med 30, 473-479.

111. Salamone F, Galvano F, Cappello F, et al. (2012) Silibinin modulates lipid homeostasis and inhibits nuclear factor kappa B activation in experimental nonalcoholic steatohepatitis. Transl Res 159, 477-486.

112. Hajiaghamohammadi AA, Ziaee A, Oveisi S, et al. (2012) Effects of metformin, pioglitazone, and silymarin treatment on non-alcoholic fatty liver disease: a randomized controlled pilot study. Hepat Mon 12, e6099.

113. Cacciapuoti F, Scognamiglio A, Palumbo R, et al. (2013) Silymarin in non alcoholic fatty liver disease. World $J$ Hepatol 5, 109-113.

114. Mourelle M, Muriel P, Favari L, et al. (1989) Prevention of $\mathrm{CCl}_{4}$-induced liver cirrhosis by silymarin. Fundam Clin Pharmacol 3, 183-191.

115. Sakata R, Nakamura T, Torimura T, et al. (2013) Green tea with high-density catechins improves liver function and fat infiltration in non-alcoholic fatty liver disease (NAFLD) patients: a double-blind placebo-controlled study. Int $J$ Mol Med 32, 989-994.

116. Bruno RS, Dugan CE, Smyth JA, et al. (2008) Green tea extract protects leptin-deficient, spontaneously obese mice from hepatic steatosis and injury. J Nutr 138, 323-331.

117. Park HJ, DiNatale DA, Chung MY, et al. (2011) Green tea extract attenuates hepatic steatosis by decreasing adipose lipogenesis and enhancing hepatic antioxidant defenses in ob/ob mice. J Nutr Biochem 22, 393-400.

118. Park HJ, Lee JY, Chung MY, et al. (2012) Green tea extract suppresses NF- $\kappa \mathrm{B}$ activation and inflammatory responses in diet-induced obese rats with nonalcoholic steatohepatitis. J Nutr 142, 57-63.
119. Masterjohn C \& Bruno RS (2012) Therapeutic potential of green tea in nonalcoholic fatty liver disease. Nutr Rev $\mathbf{7 0}$, $41-56$.

120. Jin X, Zheng RH \& Li YM (2008) Green tea consumption and liver disease: a systematic review. Liver Int $\mathbf{2 8}$, 990-996.

121. Torres-Duran PV, Paredes-Carbajal MC, Mascher D, et al. (2006) Protective effect of Arthrospira maxima on fatty acid composition in fatty liver. Arch Med Res 37, 479-483.

122. Fujimoto M, Tsuneyama K, Fujimoto T, et al. (2012) Spirulina improves non-alcoholic steatohepatitis, visceral fat macrophage aggregation, and serum leptin in a mouse model of metabolic syndrome. Dig Liver Dis 44, 767-774.

123. Ferreira-Hermosillo A, Torres-Duran PV \& Juarez-Oropeza MA (2010) Hepatoprotective effects of Spirulina maxima in patients with non-alcoholic fatty liver disease: a case series. J Med Case Rep 4, 103.

124. Dunn W, Xu R \& Schwimmer JB (2008) Modest wine drinking and decreased prevalence of suspected nonalcoholic fatty liver disease. Hepatology 47, 1947-1954.

125. Mohan V, Farooq S, Deepa M, et al. (2009) Prevalence of non-alcoholic fatty liver disease in urban south Indians in relation to different grades of glucose intolerance and metabolic syndrome. Diabetes Res Clin Pract 84, 84-91.

126. Singh SP, Nayak S, Swain M, et al. (2004) Prevalence of nonalcoholic fatty liver disease in coastal eastern India: a preliminary ultrasonographic survey. Trop Gastroenterol 25, 76-79.

127. Amarapurkar DN, Hashimoto E, Lesmana LA, et al. (2007) How common is non-alcoholic fatty liver disease in the Asia-Pacific region and are there local differences? J Gastroenterol Hepatol 22, 788-793. 